\title{
A case of a 49-year-old woman with IgG4 anti- Neurofascin 155 antibody-positive CIDP in both serum and cerebrospinal fluid
}

\section{Linlu He ( $\sim 529894996 @ q q . c o m$ )}

The Affiliated Hospital of Southwest Medical University https://orcid.org/0000-0002-6567-9590

\section{Xiu Chen}

Department of Neurology, The Affiliated Hospital of Southwest Medical University

Huan Lin

Department of Neurology,The Affiliated Hospital of Southwest Medical University

Ping Yuan

Department of Neurology,The Affiliated Hospital of Southwest Medical University

\section{Case report}

Keywords: CIDP NF155 in serum and CSF disabling tremor ataxia

Posted Date: May 23rd, 2019

DOI: https://doi.org/10.21203/rs.2.9764/v1

License: (c) (i) This work is licensed under a Creative Commons Attribution 4.0 International License.

Read Full License 


\section{Abstract}

Background CIDP (chronic inflammatory demyelinating polyneuropathy) is an autoimmune disorder of peripheral nervous system, causing demyelination and even axonal degeneration. Recently, antiNeurofascin155 (NF155) antibody-positive CIDP began to cut a figure. This is a special type of CIDP that progressively worsen and predominantly involving distal limbs. IgG4 anti- NF155 antibody-positive CIDP combining serum with CSF in clinical is very rare. The article displayed the clinical practice to evoke the diagnosis of IgG4 anti- NF155 antibody-positive CIDP in front of the disabling tremor and ataxia. We described a rare clinical disorder of IgG4 anti- NF155 antibody-positive CIDP with reviewing some of literatures. Case presentation A 49-year-old woman presented with an evaluation of weakness, numbness and a little ache of all her limbs for half a year. She revealed ataxia, intention tremor and wide-based gait. Cerebrospinal fluid (CSF) results showed the noticeably elevated protein level without pleocytosis. The findings of peripheral nerve conduction $\triangle N C V$ indicated marked prolongation of distal and F-wave latencies which conformed to demyelinating polyneuropathy. And her spinal MRI illustrated a thickened right nerve root of sacrum 1-2. IgG4 NF155 antibodies of serum together with CSF were positive by indirect immunofluorescence based on NF155 transfection cells. A high-dosage mythylprednisolone pulse followed by oral prednisolone improved her symptoms. Conclusion Disabling tremor, ataxia, younger onset age, high protein levels in CSF, marked prolongation of distal and F-wave latencies, the roots or plexus of neck and lumbosacral nerves thickening on MRI, and poor response to IVIg may consider as biomarkers of IgG4 anti- NF155 antibody-positive CIDP.

\section{Background}

CIDP was initially recognized as chronic inflammatory polyradiculoneuropathy by Dyck and colleagues in 1975, but cases consistent with probable CIDP were first described as early as 1958. [8-10] According to the reported data, the incidence of CIDP ranges from 0.7 to 1.6 cases per 100,000 persons per year, and the overall prevalence was estimated at 4.8 to 8.9 cases per 100,000 persons [11-12]. Furthermore, IgG4 anti-NF155 antibody-positive CIDP, a special type of CIDP comprises only 7 percent of all types $[1,4]$. IgG4 anti-NF155 antibody-positive CIDP in clinical, especially in both serum and CSF is very rare.In addition, NF155 is located at the paranode and expressed by the terminal loops of myelin. The primary role of IgG4 anti-NF155 antibodies may be blockade of interactions between NF155 and Caspr/contactin-1, leading to conduction failure. [5] Recent studies have suggested CIDP gradually gave the priority to disabling tremor and ataxia, which were the key clinical features of IgG4 anti-NF155 antibody-positive CIDP compared to CIDP with negative IgG4 anti-NF155 antibody. [3] The mechanism of marked sensory ataxia and tremor in anti-NF155 antibody-positive CIDP patients is still unclear.

we describe a case of a 49-year-old woman with IgG4 anti-NF155 antibody-positive CIDP. The pathogenesis, clinical manifestation diagnosis, and treatment of IgG4 anti-NF155 antibody-positive CIDP were discussed by reporting this case and reviewing the relative references.

\section{Case Presentation}


A 49-year-old woman was admitted to our ward by her fluctuating limb disorder. About half a year ago, her limbs began to feel weakness, numbness and a little ache. On admission, the neurological examination revealed characteristics of polyneuropathy, including diffuse weakness, distal symmetrical numbness with areflexia in all limbs. In addition, ataxia with a wide-based gait (video 6) was found in both lower and upper limbs, and postural as well as intention tremor were displayed in bilateral fingers (video 4 and 5). The results of Finger-to-nose test (video 1), heel-knee-tibia test (video 2) and Romberg's sign (video 3) were positive. The muscle strength of the patient's extremities was grade 4.

Brain magnetic resonance imaging (MRI) demonstrated no abnormal signals, while spinal MRI illustrated a thickened right nerve root of sacrum 1-2. (Figure1-2). Her cerebrospinal fluid (CSF) showed an extremely high protein level. The results of CSF were as follows. Protein concentration of the first time (2018-03-19) was $2.773 \mathrm{~g} / \mathrm{L}$, and the second time (2018-06-17) was $1.995 \mathrm{~g} / \mathrm{L}$, while the normal level was 150-400 $\mathrm{mg} / \mathrm{L}$. By indirect immunofluorescence on the strength of NF155 transfection cells, we captured that both the results of IgG4 anti-NF155 antibodies of serum and CSF were positive (Figure3-6). Here the description of NCV in detail. Bilateral median nerves motion transmission showed prolonged distal motor latencies, decreased conduction velocities, and low amplitudes (Figure7-10); Stimulating the elbow of the right median nerve could see a temporal dispersion wave; Bilateral tibial nerves motion transmission did not draw forth exact waves. F-wave of the left median nerve showed prolonged distal latencies, while Fwave of right median nerve and bilateral tibial nerves were not elicited (Figure11).

she was identified IgG4 anti-NF155 antibody-positive CIDP. Intravenous immunoglobulin (IVIg) was first used for her treatment, which responded poorly. When her IgG4 anti-NF155 antibodies in serum and CSF were positive, she was given a high-dosage mythylprednisolone (prednisolone,po,)pulse treatment.16 days later, her disabling tremor and ataxia symptoms improved significantly.

\section{Discussion}

CIDP is a heterogenous disorder with typical and atypical phenotypes that may share heterogenous pathogenesis. [8] Interestingly, anti-NF155 CIDP shows distinct pathological featuresdiffering from other typical CIDP. NF155 is a member of L1 family of adhesion molecules. NF155 locates in the paranodal loops of demyelinating Schwann cells and associates with the axonal cell adhesion molecules CNTN1 and contactin-associated protein-1(caspr1). [2] In addition, NF155 is one of the targets of the immune response of CIDP, playing a major role in maintaining the structure and function of Ranvier. Therefore, losing of NF155 will lead to serious demyelinating neuropathy and Purkinje neuron degeneration. Then disabling characteristic tremor (high amplitude, low frequency, postural and intention) and ataxia come to appearing on patients, [3] exactly as the woman of this case report.

Except for disabling characteristic tremor and ataxia, IgG4 anti-NF155 antibody-positive CIDP also has other typical clinical features. Compared with other types of CIDP, patients with IgG4 anti-NF155 antibodies significantly associate with younger onset age around of 20-30 years instead of 50-60, [5] Besides, the protein concentration in CSF is higher than normal values. [5] The nerve electrophysiology 
suggests marked prolongation of distal and F-wave latencies. [5] All of these symptoms were observed in the woman in case report. Furthermore, one distinguishing feature is that the roots or plexus of neck and lumbosacral nerves thickening on MRI can be found in most cases. [2-3,5] The woman in this case also showed a thickened right nerve root of sacrum 1-2.

The diagnosis of CIDP still relies on a combination of clinical and electrophysiological criteria. [1] Based on these conditions, when IgG4 anti-NF155 antibodies were detected in serum or CSF, would you can diagnose IgG4 anti-NF155 antibody-positive CIDP. Corticosteroids, intravenous immunoglobin , and/or plasmapheresis can provide short-term benefits.[6] Maintenance therapy with IVIg can induce sustained remission, increase quality of life and prevent further axonal loss.[6] The reason why IVIg can be used to treat CIDP is that it can block the Fc part of antibody and inhibit the complement pathway.[7] However, IgG4 anti-NF155 antibody-positive CIDP poor response to IVIg .[3] IgG4 antibodies do not bind to C1q and have low affinity to Fc receptors and complement.[1,3] Therefore, the treatment of IgG4 anti-NF155 antibody-positive CIDP mainly depends on corticosteroids.

\section{Conclusion}

IgG4 anti-NF155 antibody-positive CIDP in serum and CSF in clinical is very rare. Disabling characteristic tremor (high amplitude, low frequency, postural and intention) and ataxia are the symbolic symptoms of this disorder. Meanwhile, younger onset age, high protein levels in CSF, marked prolongation of distal and F-wave latencies, the roots or plexus of neck and lumbosacral nerves thickening on MRI, and poor response to IVIg are other important clinical characteristics. All of these traits may be considered as a biomarker of IgG4 anti-NF155 antibody-positive CIDP. The therapy of IgG4 anti-NF155 antibody-positive CIDP primarily relies on corticosteroids. IVIg have poor effect on it.

\section{Abbreviations}

CIDP: chronic inflammatory demyelinating polyneuropathy; CSF: Cerebrospinal fluid; NCV『nerve

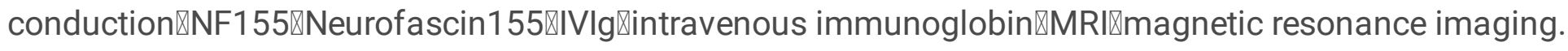

\section{Declarations}

Ethics approval and consent to participate:

This study is accordance with the Declarations of Helsinki. This report has been approved by the Ethics Board of The Affiliated Hospital of Southwest Medical University.All authors agreed the publish statements of BMC Neurology.

Consent for publication

Written informed consent was obtained from the patient for publication of this Case report and any accompanying images. A copy of the written consent is available for review by the Editor-in-Chief of this 
journal.

Availability of data and material

All data generated or analyzed during this study are included in this published article and its supplementary information files.

Competing interest

The author declare that they have no competing interests.

Funding

Not applicable.

Authors' contributions

YP introduced the patient to this study. HLL carried out the study, collected important background information and drafted the manuscript. HLL, CX, LH, and YP participated in tits design and coordination and helped to draft the manuscript. All authors read the final manuscript.

Acknowledgements

We thank The Affiliated Hospital of Southwest Medical University for making acquisition and analysis of all the auxiliary examination data, and for making the data available to us.

\section{References}

1. Mathey EK, Park SB, Hughes RA, et al. Chronic inflammatory demyelinating polyradiculoneuropathy: from pathology to phenotype. Journal of neurology, neurosurgery, and psychiatry 2015; 86(9): $973-85$.

2. Devaux JJ, Miura Y, Fukami Y, et al. Neurofascin-155 IgG4 in chronic inflammatory demyelinating polyneuropathy. Neurology 2016; 86(9): 800-7.

3. Querol L, Nogales-Gadea G, Rojas-Garcia R, et al. Neurofascin IgG4 antibodies in CIDP associate with disabling tremor and poor response to IVIg. Neurology 2014; 82(10): 879-86.

4. Ng JK, Malotka J, Kawakami N, et al. Neurofascin as a target for autoantibodies in peripheral neuropathies. Neurology 2012; 79(23): 2241-8.

5. Ogata $\mathrm{H}$, Yamasaki R, Hiwatashi A, et al. Characterization of IgG4 anti-neurofascin 155 antibodypositive polyneuropathy. Annals of clinical and translational neurology 2015; 2(10): 960-71.

6. Dalakas MC. Advances in the diagnosis, pathogenesis and treatment of CIDP. Nature reviews Neurology 2011; 7(9): 507-17. 
7. Sudo M, Yamaguchi Y, Spath PJ, et al. Different IVIG glycoforms affect in vitro inhibition of antiganglioside antibody-mediated complement deposition. PloS one 2014; 9(9): e107772.

8. Ryan M, Ryan SJ. Chronic inflammatory demyelinating polyneuropathy: considerations for diagnosis, management, and population health. The American journal of managed care 2018; 24(17 Suppl): S371s9.

9. Dyck PJB, Tracy JA. History, Diagnosis, and Management of Chronic Inflammatory Demyelinating Polyradiculoneuropathy. Mayo Clinic proceedings 2018; 93(6): 777-93.

10. Dyck PJ, Lais AC, Ohta M, Bastron JA, Okazaki H, Groover RV. Chronic inflammatory polyradiculoneuropathy. Mayo Clinic proceedings 1975; 50(11): 621-37.

11. Rajabally YA, Simpson BS, Beri S, Bankart J, Gosalakkal JA. Epidemiologic variability of chronic inflammatory demyelinating polyneuropathy with different diagnostic criteria: study of a UK population. Muscle \& nerve 2009; 39(4): 432-8.

12. Laughlin RS, Dyck PJ, Melton LJ, 3rd, Leibson C, Ransom J, Dyck PJ. Incidence and prevalence of CIDP and the association of diabetes mellitus. Neurology 2009; 73(1): 39-45.

\section{Figures}

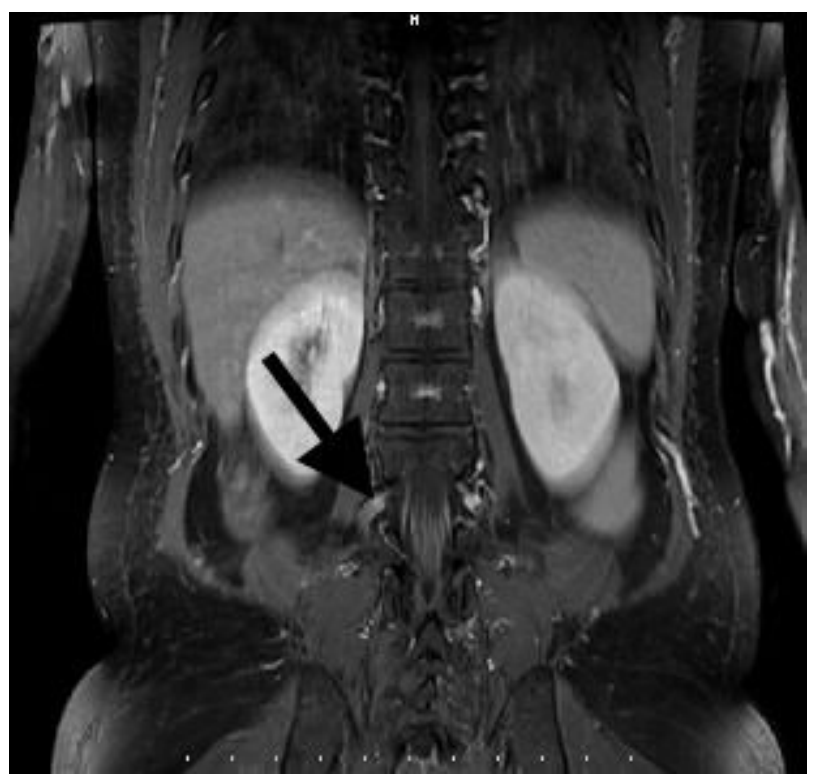

\section{Figure 1}

spinal MRI with thickened nerve roots 


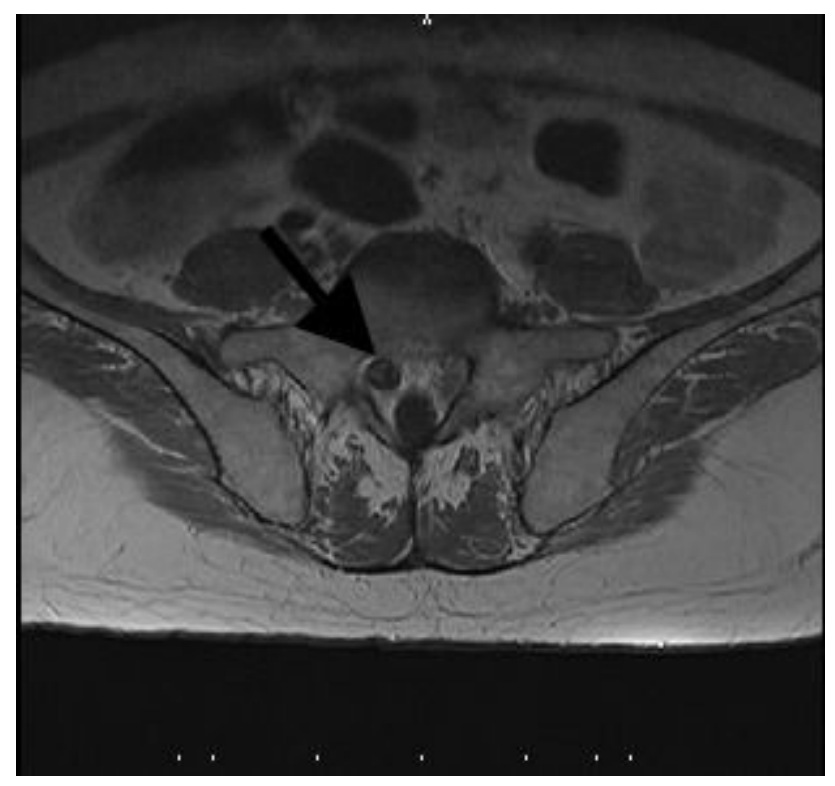

Figure 2

spinal MRI with thickened nerve roots

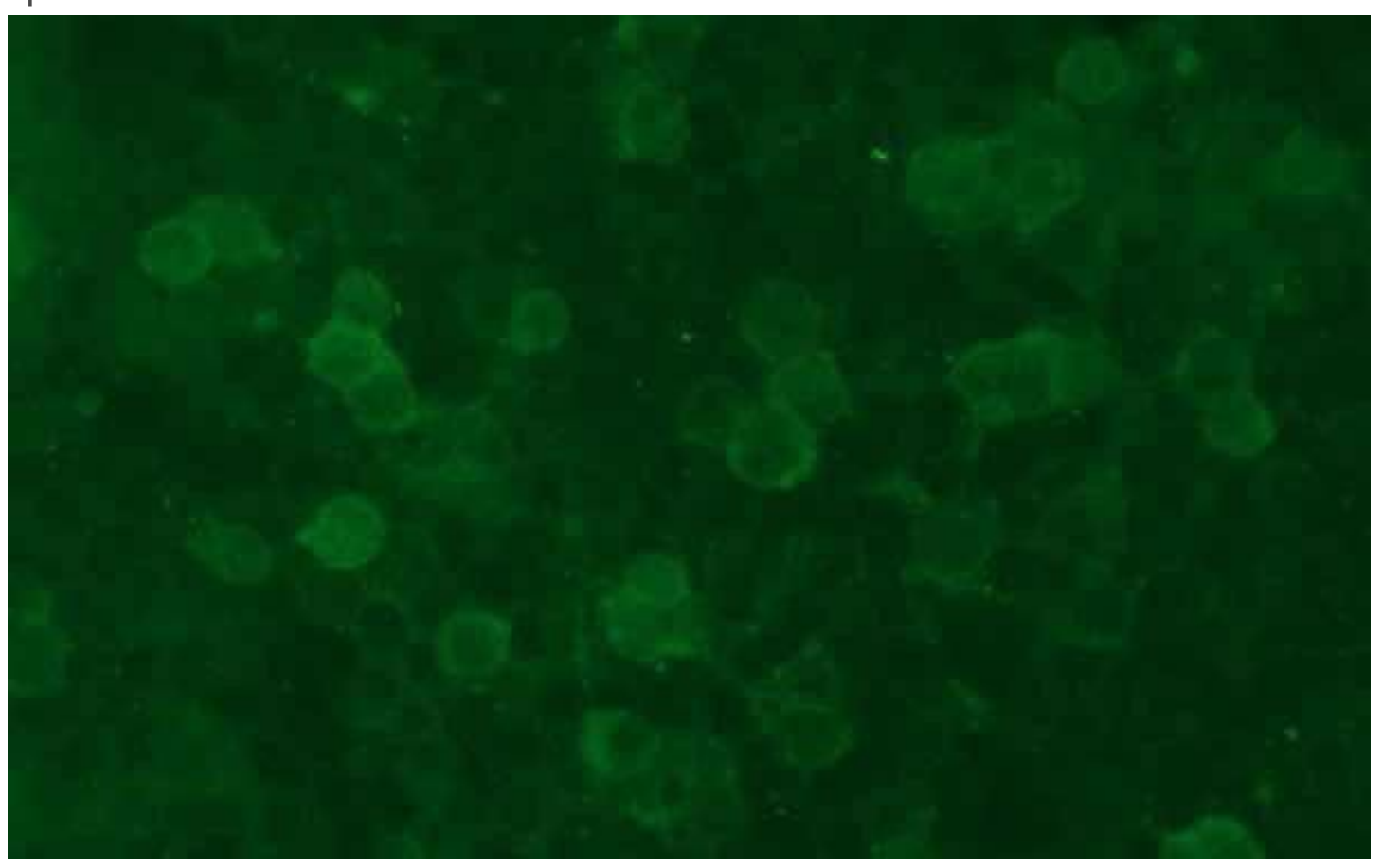

Figure 3

indirect immunofluorescence based on NF155 transfection cells of serum, NF155 IgG4, anti-NF155 IgG4 antibodies: 1:32 


\section{Figure 4}

indirect immunofluorescence based on NF155 transfection cells of serum, control group

\section{Figure 5}

indirect immunofluorescence based on NF155 transfection cells of CSF, NF155 IgG4, anti-NF155 IgG4 antibodies: 1:3.2 


\section{Figure 6}

indirect immunofluorescence based on NF155 transfection cells of CSF, control group

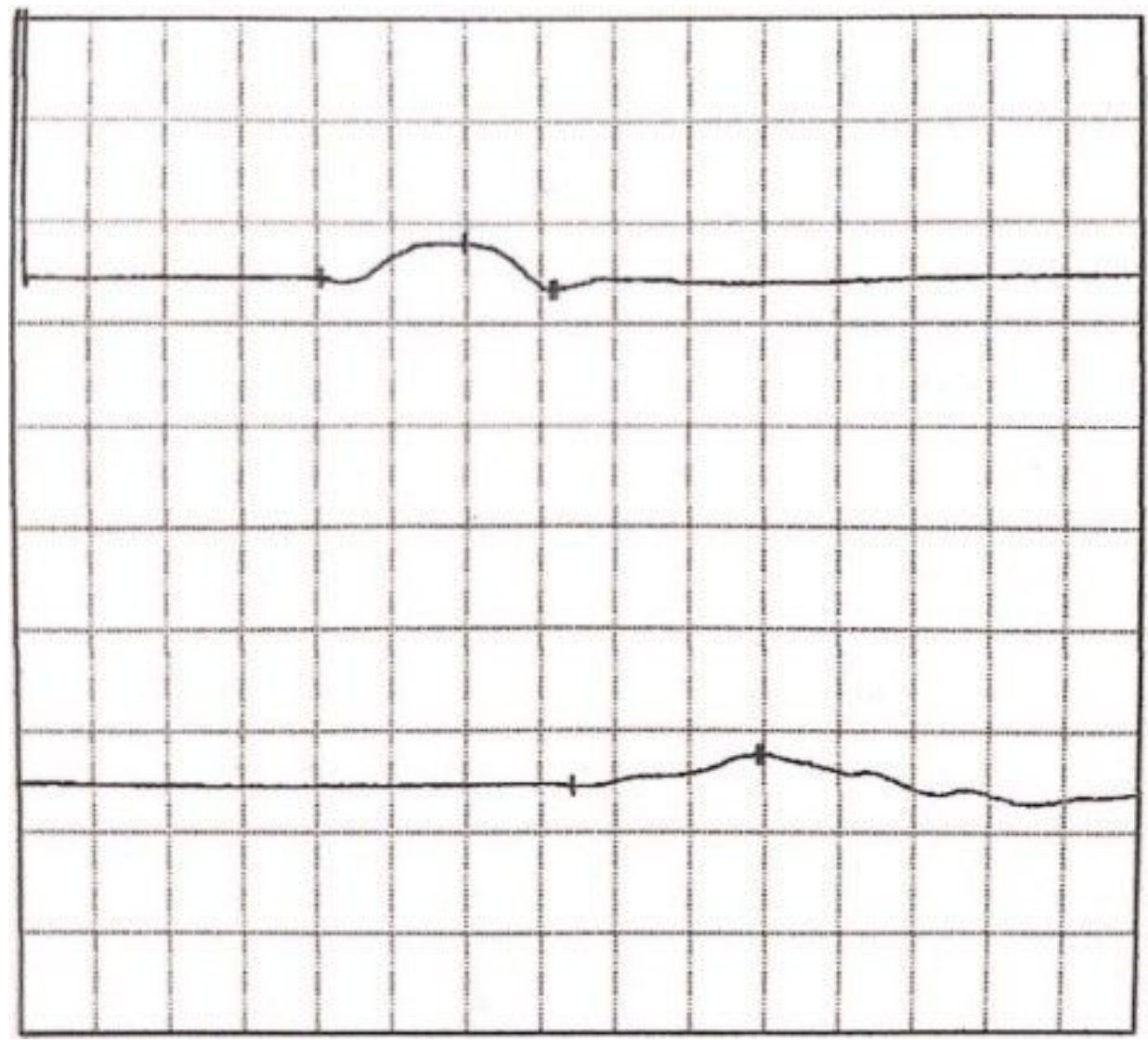

Figure 7 
the right median nerve MCS

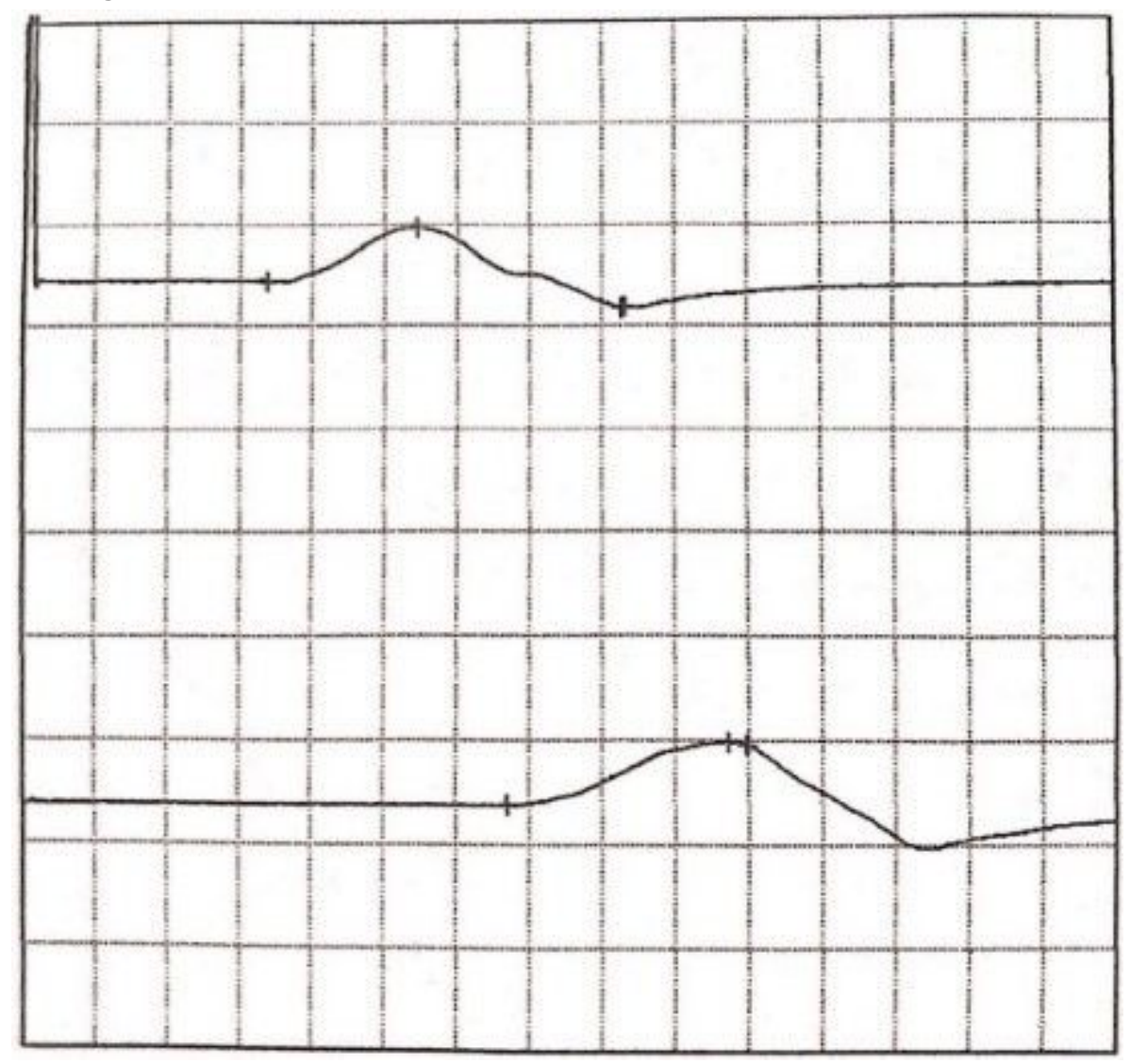

Figure 8

the left median nerve MCS 


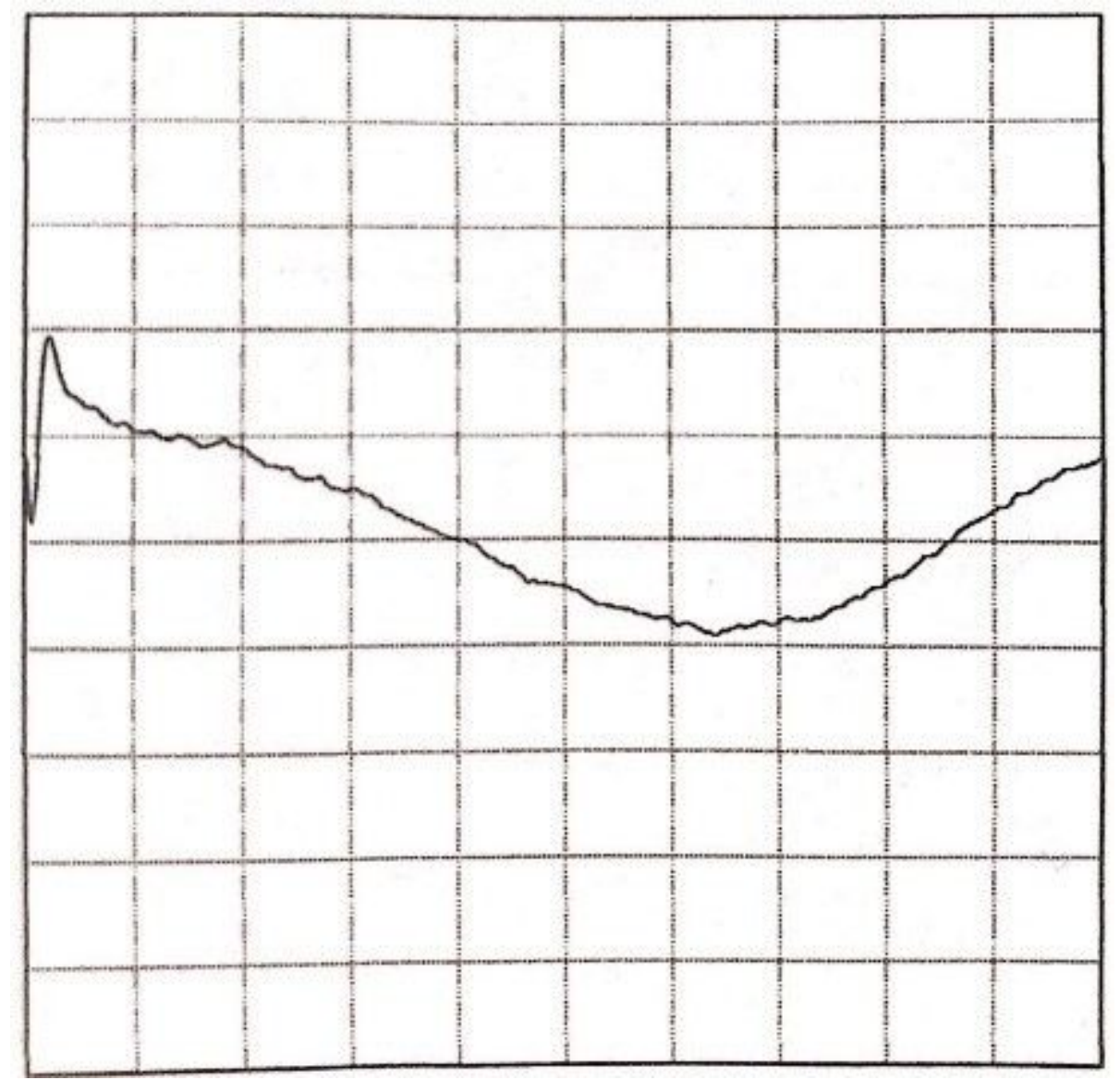

Figure 9

the right median nerve SCS 


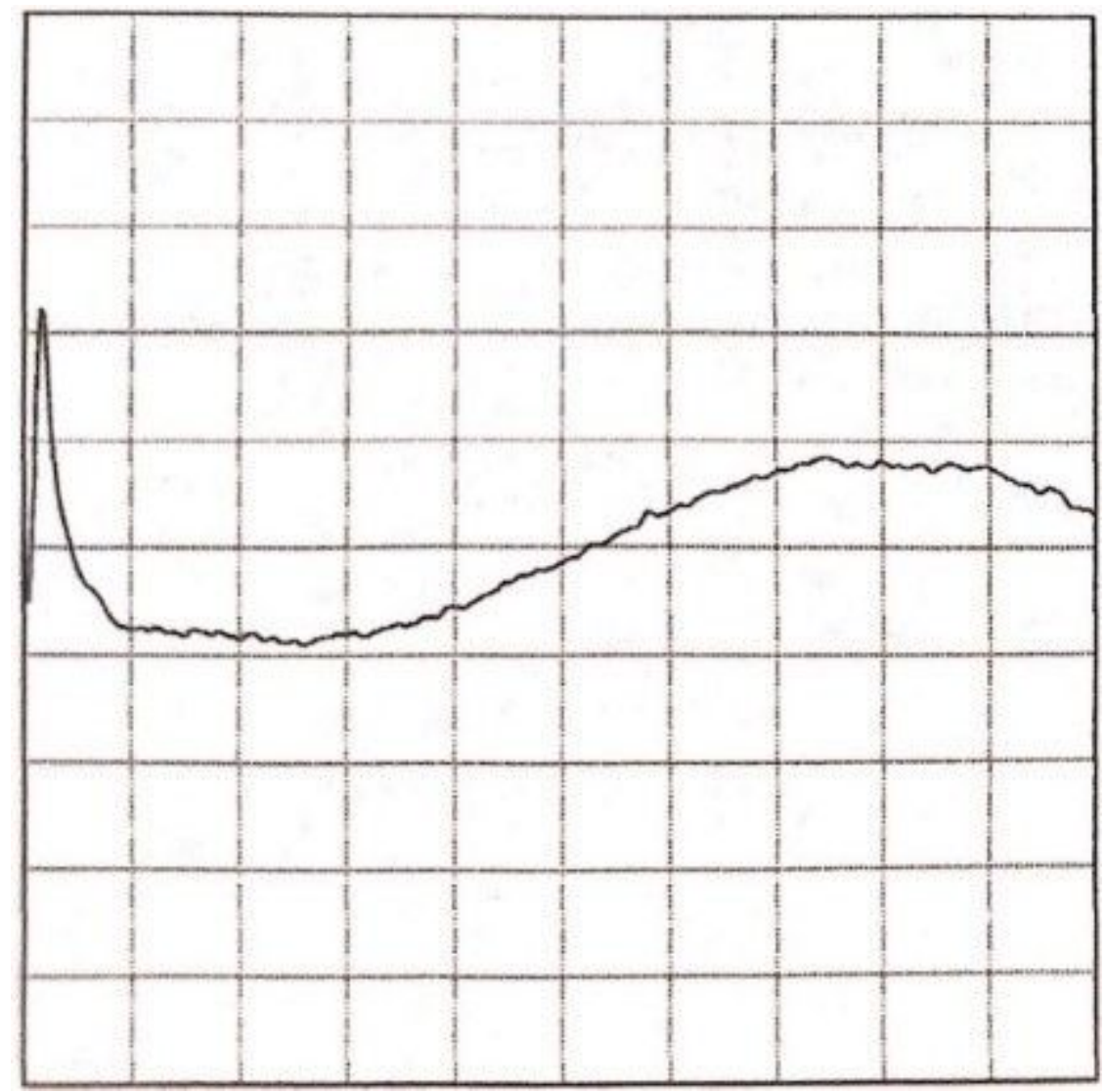

Figure 10

the left median nerve SCS 


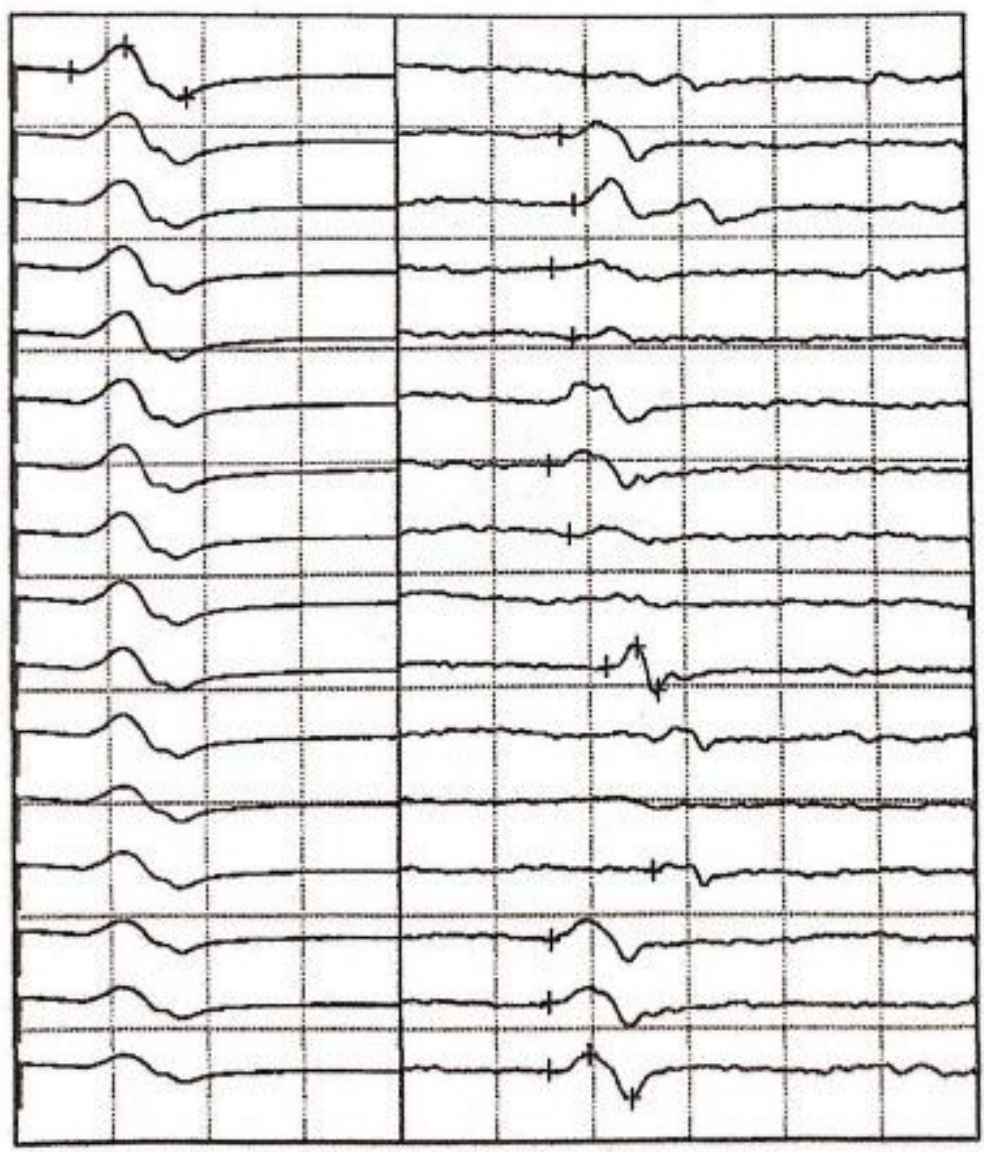

Gain 5mV,500uV Analy. $10 \mathrm{~ms}$

Figure 11

F-wave of the left median nerve

\section{Supplementary Files}

This is a list of supplementary files associated with this preprint. Click to download.

- additionalfile13video2heelkneetibiatest.mp4

- carechecklist.docx

- additionalfile15video4intentiontremorofupperlimbs.mp4

- additionalfile16.MP4

- additionalfile17.MP4

- additionalfile14.MP4

- additionalfile12video1Fingertonosetest.MOV 